\title{
Comparación de los casos de neumonía en niños durante la pandemia de influenza AH1N1 (pH1N1) en un Hospital Pediátrico del Perú
}

\section{Comparison of pneumonia cases in children during pandemic of influenza AHINI (pH1N1) in a Pediatric Hospital of Peru}

\author{
Edwin Miranda-Choque ${ }^{1}$, Sonia Farfán-Ramos ${ }^{2}$, Carmen Injante-Estrada ${ }^{3}$ \\ http://dx.doi.org/10.21503/CienciayDesarrollo.2014.v17i1.04
}

\section{RESUMEN}

Durante la pandemia del pH1N1 se atendieron casos severos de neumonías por diferentes etiologías, por lo que nosotros quisimos describir y comparar las características clínicas y laboratoriales de los casos de neumonías positivas y negativas al $\mathrm{pH} 1 \mathrm{~N} 1$.

Estudio trasversal analítico, se revisaron las historias clínicas de los niños hospitalizados por neumonía entre los meses de junio a octubre del 2009 en el Instituto Nacional de Salud del Niño con exámenes virológicos de tr-PCR TR para $\mathrm{pH} 1 \mathrm{~N} 1$ e infiltrado pulmonares en sus radiografías.

Estudiamos a 187 casos de neumonía, de los cuales 50 fueron neumonía adquirida en la comunidad (NAC) positivas al $\mathrm{pH} 1 \mathrm{~N} 1$ y 24 fueron como neumonía nosocomial (NN) positivas al $\mathrm{pH} 1 \mathrm{~N} 1$, de ellos fallecieron 12; todos presentaron factores preexistentes. Los casos de NAC positivos al $\mathrm{pH} 1 \mathrm{~N} 1$ tuvieron más presencia de fiebre $(\mathrm{p}<0,005)$ y linfopenia $(\mathrm{p}<0,005)$ que los casos de neumonía negativos al $\mathrm{pH} 1 \mathrm{~N} 1$, pero menos presencia de condiciones preexistentes $(\mathrm{p}<0,001)$ y menor estadía hospitalaria $(\mathrm{p}<0,005)$.

Los casos severos de NAC por $\mathrm{pH} 1 \mathrm{~N} 1$ con $\mathrm{PCR} \geq 10 \mathrm{mg} / \mathrm{L}$ tienen un riesgo elevado con un OR de $4,2(1,5-6,8) \mathrm{p}<0,005$. Los casos de NN tuvieron mayor probabilidad de fallecer.

Los resultados de este estudio mostraría que los signos, síntomas y exámenes de laboratorio ayudan muy poco en la diferenciación de la NAC negativas o positivas al pH1N1; el PCR elevado y la presentación de condición preexistente están asociados a casos severos de NAC al pH1N1.

Palabras clave: $p H 1 N 1$, influenza, neumonia, niños.

\section{ABSTRACT}

During the pH1N1 pandemic were treated severe cases of pneumonia by different etiologies, so we wanted to describe and compare the clinical and laboratory features of positive and negative pneumonia cases to the pH1N1.

Transversal analytical study, it was reviewed the medical histories of the children hospitalized for pneumonia between the months of June to October, 2009 at Instituto Nacional de Salud del Niño (Child Health National Institute) with tr-CRP TR virological tests for $\mathrm{pH} 1 \mathrm{~N} 1$ and pulmonary infiltrate in their $\mathrm{x}$-rays.

We studied 187 pneumonia cases, of which 50 were positive the $\mathrm{pH} 1 \mathrm{~N} 1$ community-acquired pneumonia and 24 were as positive pneumonia in hospital to the $\mathrm{pH} 1 \mathrm{~N} 1$, of them died 12; all had pre-existing

\footnotetext{
Médico Infectólogo. Instituto Nacional de Salud del Niño y docente de la EAP Medicina Humana de la UAP. E-mail: emirandach@hotmail.com

Enfermera especialista en pediátrica, Magister en Salud Pública y Comunitaria. Hospital 2 de Mayo. Lima, Perú. E-mail: soniaf42@hotmail.com

Enfermera especialista en cuidados intensivos. E-mail: carmenneussa@gmail.com
} 
factors. Positive cases of community-acquired pneumonia to the $\mathrm{pH} 1 \mathrm{~N} 1$ had more presence of fever $(\mathrm{p}<$ $0.005)$ and lymphopenia $(\mathrm{p}<0.005)$ than negative pneumonia cases to the $\mathrm{pH} 1 \mathrm{~N} 1$, but less presence of pre-existing conditions $(\mathrm{p}<0.001)$ and less hospital stay $(\mathrm{p}<0.005)$.

Severe cases of community-acquired pneumonia by $\mathrm{pH} 1 \mathrm{~N} 1$ with CRP $\geq 10 \mathrm{mg} / \mathrm{L}$ have a high risk with a 4.2 OR $(1,5-6,8) \mathrm{p}<0,005$. Pneumonia in hospital cases were more likely to die.

The results of this study would show that the signs, symptoms and lab tests help very little in the differentiation of the negative or positive community-acquired pneumonia to the $\mathrm{pH} 1 \mathrm{~N} 1$; the elevated $\mathrm{CRP}$ and the presentation of pre-existing condition are associated with severe cases of community-acquired pneumonia to the $\mathrm{pH} 1 \mathrm{~N} 1$.

Key words: $p H 1 N 1$, influenza, pneumonia, children.

\section{INTRODUCCIÓN}

Lapandemia del virus de influenzaAH1N1/2009 pandémico ( $\mathrm{pH} 1 \mathrm{~N} 1)$, declarado así por la Organización Mundial de la Salud (OMS) (1), fue ocasionada por un virus con una nueva variante por recombinación genética con elementos porcinos, aviarios y humanos(2).

Los primeros casos ocurrieron a mediados de abril del 2009 al sur de EEUU y en Oaxaca México (3-6), extendiéndose posteriormente a nivel mundial (7), por lo que la OMS e instituciones internacionales y nacionales elaboraron medidas frente a la pandemia $(8,9)$, el Ministerio de Salud del Perú (MINSA), informó el primer caso adulto de pH1N1 el 09 de mayo 2009, (10) y una semana después el primer caso pediátrico atendido en el INSN (Instituto Nacional de salud del niño), ambos procedentes de Lima.

Estudios iniciales en niños hospitalizados por neumonía por $\mathrm{pH} 1 \mathrm{~N} 1$ reportaron tasas mayores que las ocasionadas por la influenza estacional (11). Ambos con espectro clínico muy parecido que cursaron con tos, alza térmica, cefalea, malestar general, artralgias, mialgias, rinorrea, dolor de garganta, disnea, roncantes, sibilantes crepitantes. Atribuyendo a los casos con pH1N1 mayores síntomas gastrointestinales como diarrea o vómitos, reportándose casos severos como complicaciones neurológicas, hematológicas e insuficiencia respiratoria $(12,13)$; Posteriormen- te se reportan diferentes formas de presentación en todas partes del mundo (14); por lo que se plantea que el espectro clínico de la infección el pH1N1 puede estar relacionado con la etnicidad y el estado inmunológico del huésped $(14,15)$. Por lo que es necesario ampliar información de esta enfermedad en diferentes regiones.

El presente estudio tiene como objetivo describir y comparar las características clínicas, demográficas y laboratoriales de los casos hospitalizados por neumonía positiva y negativa al $\mathrm{pH} 1 \mathrm{~N} 1$ en un hospital pediátrico durante la pandemia del pH1N1.

\section{POBLACIÓN Y MÉTODO}

Se realizamos un estudio retrospectivo trasversal analítico. La población estaba constituido por todos los pacientes con neumonía provenientes de la comunidad que fueron hospitalizados en el INSN en el periodo junio a octubre 2009, meses de mayor actividad de la pandemia en el Perú $(10,16)$. Los casos se identificaron de la oficina de estadísticas del INSN, los criterios de inclusión fueron pacientes con síntomas respiratorios de presentación aguda, presencia de infiltrado pulmonar en su radiografía de tórax, con examen virológico por tr-PCR TR al pH1N1 en el INS (Instituto Nacional de Salud), con el criterio de exclusión de historias clínicas incompletas. Con la muestra obtenida se agruparon en 2 sub poblaciones, la primera los casos de neumonía ad- 
quiridos en la comunidad (NAC) positivos al pH1N1 (NAC (+) pH1N1) y la segunda los casos de NAC negativos al pH1N1 (NAC (-) pH1N1) considerados como neumonía por otra causa. Se consideró como muestra especial a los casos de neumonía nosocomial (NN) positivos al pH1N1 (NNpH1N1), si los niños iniciaron los síntomas respiratorias 72 horas después de su ingreso hospitalario. Se definió también severidad por neumonía a todo caso con saturación de oxigeno menos de $90 \%$ medido por oxímetro de pulso.

Se recolectó la información demográfica y clínica de los casos de NAC, que incluía variables como edad, sexo, tiempo de enfermedad, saturación menor $90 \%$ de $\mathrm{O}_{2}$ al ingreso hospitalario (hipoxemia), estado nutricional mediante el software WHO Anthro (versión 3.2.2, Geneva, Switzerland); datos demográficos, condición preexistentes o comorbilidad, como déficit neurológico (portador de sistema de derivación interna o externa, parálisis cerebral infantil, retardo del desarrollo psicomotriz), cardiopatía congénita (diagnostico mediante ecocardiografía), infección del virus de inmunodeficiencia Humana (VIH) (confirmado virológicamente), síndrome de obstrucción bronquial aguda (SOBA) o asma; además, valores bioquímicos de proteína $\mathrm{c}$ reactiva (PCR) al ingreso hospitalario, características del hemograma al ingreso hospitalario como leucopenia (leucocitos menos de 4000 por ml.), leucocitosis (leucocito más de 10000 por $\mathrm{ml}$.), linfopenia (menos de 1500 por ml. para niños más de 3 años y menos de 3 000 por $\mathrm{ml}$. para los niños menores de 3 años), trombocitosis (plaquetas más de 450000 por $\mathrm{ml}$.), patrón radiográfico de pulmón al ingreso hospitalario evaluado por medico radiólogo con más de 10 años de experiencia, insuficiencia respiratoria, etc. De los casos NNpH1N1, solo se recolectaron de datos demográficos y condiciones pre existente.
Todos los procedimientos del presente estudio tratan de preservar la integridad y los derechos fundamentales de los pacientes sujetos a investigación, de acuerdo a los lineamientos de las buenas prácticas y de ética en investigación biomédica, por lo que obtuvo aprobación del comité de ética del INSN.

\section{Análisis Estadístico}

Por medio de software estadístico STATA la versión 10, se realizó un análisis descriptivo de las variables continuas o discretas. Para las variables continuas se realizó análisis de tendencia central y para variables discretas análisis de frecuencia. Se comparó los casos de NAC (+) pH1N1 y NAC $(-)$ pH1N1, mediante un análisis bivariado, con las variables continuas se uso t de Student o U de Mann Whitney y análisis de U de Kruskal Wallis de acuerdo a su distribución normal, para las variables discretas se realizó el Chi2 cuadrado o prueba exacta de Fisher. Se realizó un análisis de exploratorio de riesgo mediante OR para identificar los factores de riesgo para neumonía severa o muerte. Se comparó también los casos NAC (+) pH1N1 con NNpH1N1. Se consideró una diferencia significativa un valor de $\mathrm{p}$ de $<0.05$.

\section{RESULTADOS}

Entre junio y octubre del 2009 se hospitalizaron 432 pacientes con diagnóstico infecciones respiratoria aguda, De ellos 171 fueron casos de neumonía, de los cuales 50 fueron NAC (+) $\mathrm{pH} 1 \mathrm{~N} 1,87$ fueron NAC (-) pH1N1 y 24 fue-

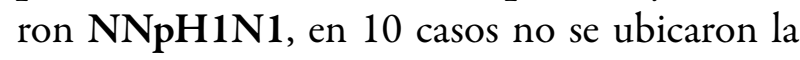
historia clínica. Los casos sumados de $\mathrm{pH} 1 \mathrm{~N} 1$ (NAC (+) pH1N1 mas NNpH1N1) presentaron, administración de oseltamivir el 77,0\% $(57 / 74)$, presentaron saturación de $0_{2}$ menos de $90 \%$, el $35,1 \%(26 / 74)$, uso de ventilador mecánico 21,6\%(16/74) el promedio de días de hospitalización fue de 13,4 (rango de 3 a 90 días), Fallecieron 12 casos, 05 casos como NAC (+) 
pH1N1 y 07 casos NNpH1N1, todos con condiciones preexistentes.

Neumonía Adquirida en la Comunidad por influenza AH1N1/2009 pandémico (NAC (+) pH1N1).

Los niños menores de 2 años fueron el $48 \%$ (24/50) y menores de 6 años el 72\% (36/50); el 78\%(39/50) tuvieron comorbilidades o condiciones pre existentes, siendo más frecuente SOBA/asma, desorden neurológico, cardiopatía y desnutrición (tabla 1). El 64\%(32/50) recibió antibióticos previamente a la hospitalización. Saturación de oxigeno menor de 90\% fueron
$16(32 \%)$, requirieron ventilador mecánico 08 (16\%), recibieron oseltamivir 41 (82\%), la mediana de tiempo de enfermedad antes de llegar al hospital fue de 5 días (rango 1 a 10 días). El promedio de días de hospitalización fue de 10 días (rango de 3 a 30 días).

Los signos y síntomas de los casos al ingreso hospitalario fueron fiebre el $90 \%$ (45/90), tos $86 \%$ $(43 / 50)$, rinorrea $60 \%(30 / 50)$, expectoración $44 \%$ (22/50), vómitos 28\% (14/50), diarrea $28 \%(14 / 50)$ (tabla $\left.n^{\circ} 01\right)$, complicaciones por pH1N1 fueron encefalitis 01 caso, otitis media 02 caso.

Tabla 1. Características de los niños hospitalizados con neumonía por pH1N1 en el INSN, LimaPerú, Junio-Setiembre, 2009.

\begin{tabular}{|c|c|c|c|c|c|}
\hline \multicolumn{2}{|c|}{ Características } & $\begin{array}{c}\text { NAC } \\
(+) \mathrm{pH}_{1 \mathrm{~N} 1} 1^{*} 50\end{array}$ & $\begin{array}{c}\text { NN } \\
\text { pH1N1** } 24\end{array}$ & Total & valor $p$ \\
\hline \multicolumn{2}{|c|}{ Hombres Sexo__ n ${ }^{\circ}(\%)$} & $24(48)$ & $14(58)$ & $38(51)$ & 0,405 \\
\hline \multirow[t]{6}{*}{ Edad__años } & Mediana & 2 & 7 & 2,5 & 0,008 \\
\hline & Rango & $0,1-15$ & $0,1-17$ & $0,1-17$ & \\
\hline & Grupo etareo__ $\mathrm{n}^{\circ}(\%)$ & & & & 0,006 \\
\hline & $<2$ años__ $\quad \mathrm{n}^{\circ}(\%)$ & $24(48)$ & $05(21)$ & $29(39)$ & \\
\hline & $2-5$ años_ $\mathrm{n}^{\circ}(\%)$ & $12(24)$ & $03(13)$ & $15(20)$ & \\
\hline & 6-18 años__ $\mathrm{n}^{\circ}(\%)$ & $14(28)$ & $16(67)$ & $30(41)$ & \\
\hline Procedencia & Lima__ $\mathrm{n}^{\circ}(\%)$ & $46(92)$ & $13(54)$ & $59(80)$ & \\
\hline \multicolumn{2}{|c|}{ Condiciones pre existentes } & $25(50)$ & $24(100)$ & & 0,000 \\
\hline & Asma/SOBA_ n ${ }^{\circ}(\%)$ & $16(32)$ & $08(33)$ & $24(32)$ & 0,090 \\
\hline & Déficit neurológico crónico.__ $\mathrm{n}^{\circ}(\%)$ & $13(26)$ & $03(13)$ & $16(22)$ & 0,154 \\
\hline & Algún grado de desnutrición & $16(32)$ & $10(40)$ & $16(22)$ & 0,608 \\
\hline & Desnutrición aguda__ $\mathrm{n}^{\circ}(\%)$ & $08(16)$ & $03(13)$ & $11(15)$ & \\
\hline & Desnutrición crónica__ $\mathrm{n}^{\circ}(\%)$ & $08(16)$ & $07(30)$ & $15(22)$ & \\
\hline & Infección respiratoria previa__ $\mathrm{n}^{\circ}(\%)$ & $07(14)$ & $06(25)$ & $13(18)$ & 0,199 \\
\hline & Sd. down/klinef.__ $\mathrm{n}^{\circ}(\%)$ & $02(04)$ & $01(08)$ & $03(04)$ & \\
\hline & $\mathrm{VIH} \_\mathrm{n}^{\circ}(\%)$ & $02(04)$ & 0 & $02(03)$ & \\
\hline & Pancitopenia/neutropenia__ $\mathrm{n}^{\circ}(\%)$ & $01(02)$ & $03(13)$ & $04(05)$ & \\
\hline & Cardiopatía congénita__ $\mathrm{n}^{\circ}(\%)$ & $02(04)$ & $04(17)$ & $06(08)$ & \\
\hline \multicolumn{6}{|c|}{ - } \\
\hline & $\mathrm{PCR} \geq 10 \mathrm{mg} / \mathrm{L} \_\mathrm{n}^{\circ}(\%)$ & $13(26)$ & $06(25)$ & $19(26)$ & 0,927 \\
\hline & Uso de oseltamivir__ $\mathrm{n}^{\circ}(\%)$ & $41(82)$ & $16(67)$ & $57(77)$ & 0,142 \\
\hline & Mortalidad__ $\mathrm{n}^{\circ}(\%)$ & $5(10)$ & $7(29)$ & $12(16)$ & 0,036 \\
\hline
\end{tabular}

${ }^{*} \mathrm{NAC}(+) \mathrm{pH} 1 \mathrm{~N} 1=$ neumonía adquirida en la comunidad por influenza AH1N1/2009 pandémico

${ }^{* *} \mathrm{NNpH} 1 \mathrm{~N} 1=$ neumonía nosocomial por influenza AH1N1/2009 pandémico 
Con respecto al hemograma, leucopenia presento el $14 \%(07 / 50)$, leucocitosis el $48 \%(24 / 50)$, linfopenia el $34 \%(17 / 50)$, trombocitosis lo presentaron el 10\% (5/50). El PCR más de $10 \mathrm{mg} / \mathrm{L}$ tuvieron el $26 \%(13 / 50)$. Con respecto a la radiografía de tórax el 48\% (24/50) presentó infiltrado en parches y el 44\% (22/50) infiltrado intersticial.

En el análisis exploratorio buscando asociación con neumonía severa, hallamos que tener la PCR mayor a $10 \mathrm{mg} / \mathrm{L}$ estaba asociado a saturación de $0_{2}$ menos $90 \%$ con un OR (Odds Ratio) de de 4,2 (IC: 1,5-6,8, p=0,001). No se encontró asociación el antecedente de asma o el uso de oseltamivir.
Neumonía Nosocomial por el nuevo virus de Influenza AH1N1/2009 pandémico (NNpH1N1).

De los 24 casos NNpH1N1, 16 contrajeron la infección en las salas de medicina, cardiología y neumología; 08 en la UCI. Los mayores de 6 años fueron el 67\%(16/24); las condiciones pre existente más frecuentes fueron desnutrición crónica, antecedentes de infecciones respiratorias, enfermedad del colágeno, cardiopatía congénita y déficit neurológico crónico. 08 casos necesitaron ventilador mecánico. Los fallecidos fueron 07 por lo que hacen una mortalidad del $29 \%$ las condiciones pre existentes fueron pancitopenia (02), cardiopatía congénita (03) Lupus Eritematoso Sistémico (01) y dermatomiositis (01).

Tabla 2. Características de los niños con NAC(-)pH1N1 y NAC(+)pH1N1 en el INSN, Lima-Perú, Junio-Setiembre, 2009.

\begin{tabular}{|c|c|c|c|}
\hline Características & NAC(-)pH1N1* & $\mathrm{NAC}(+) \mathrm{pH} 1 \mathrm{~N} 1^{* *}$ & valor $p$ \\
\hline Todos los casos & 87 & 50 & \\
\hline Hombres __ $\mathrm{n}^{\circ}(\%)$ & $42(48,3)$ & $24(48,0)$ & 0,975 \\
\hline \multicolumn{4}{|l|}{ Edad__años } \\
\hline Mediana & 2 & 2 & 0,899 \\
\hline RIQ & $0,8-07$ & $1,0-06$ & \\
\hline Grupo etareo & & & 0,222 \\
\hline$<2$ años__ $\mathrm{n}^{\circ}(\%)$ & $41(47,1)$ & $24(48,0)$ & \\
\hline $2-5$ años__ $n^{\circ}(\%)$ & $12(13,9)$ & $12(24,0)$ & \\
\hline$>$ 5años__ $\mathrm{n}^{\circ}(\%)$ & $22(39,1)$ & $14(28,0)$ & \\
\hline Lima__ $\mathrm{n}^{\circ}(\%)$ & $66(75,9)$ & $46(92,0)$ & 0,019 \\
\hline Condiciones pre existente__ $\mathrm{n}^{\circ}(\%)$ & $62(71,3)$ & $18(36,0)$ & 0,000 \\
\hline Saturación $0_{2}<90 \% \_\mathrm{n}^{\circ}(\%)$ & $33(37,9)$ & $16(32.0)$ & 0,483 \\
\hline Días de hospitalización___Mediana & 12 & 9 & 0,001 \\
\hline RIQ & $08-29$ & $05-15$ & \\
\hline Requirieron ventilación mecánica___ $\mathrm{n}^{\circ}(\%)$ & $24(27,6)$ & $8(16)$ & 0,123 \\
\hline Mortalidad__ $\mathrm{n}^{\circ}(\%)$ & $8(09,2)$ & $5(10)$ & 0,877 \\
\hline
\end{tabular}


Tabla 3. Signos y síntomas clínicos de los niños con NAC(-)pH1N1 y NAC(+) en el INSN, LimaPerú Junio-Setiembre, 2009.

\begin{tabular}{|c|c|c|c|}
\hline Signos y Síntomas & $\begin{array}{c}\text { NAC(-)pH } 1 \mathbf{N} 1^{*} \\
\mathbf{n}(\%)\end{array}$ & $\begin{array}{c}\mathrm{NAC}(+) \mathrm{pH} 1 \mathrm{~N} 1^{* *} \\
\mathbf{n}(\%)\end{array}$ & valor $p$ \\
\hline Total pacientes & 87 & 50 & \\
\hline Fiebre & $61(70,1)$ & $45(90,0)$ & 0,009 \\
\hline Tos & $76(87,4)$ & $43(86,0)$ & 0,353 \\
\hline Rinorrrea & $59(67,8)$ & $30(60,0)$ & 0,578 \\
\hline Expectoración & $43(49,4)$ & $22(44,0)$ & 0,540 \\
\hline Vómitos & $32(36,8)$ & $14(28,0)$ & 0,295 \\
\hline Diarrea & $16(18,4)$ & $14(28,0)$ & 0,190 \\
\hline Dolor abdominal & $03(03,5)$ & $04(08,0)$ & 0,478 \\
\hline Mialgia & $02(02,3)$ & $05(10,0)$ & 0,135 \\
\hline Roncantes & $35(40,2)$ & $23(46,0)$ & 0,511 \\
\hline Crepitantes & $22(25,3)$ & $16(32,0)$ & 0,332 \\
\hline Sub crepitantes & $45(51,2)$ & $25(50,0)$ & 0,846 \\
\hline Sibilancias & $35(40,3)$ & $24(48,0)$ & 0,377 \\
\hline
\end{tabular}

Tabla 4. Características laboratoriales de los niños con NAC(-)pH1N1 y NAC(+)pH1N1 en el INSN, Lima-Perú Jun.-Set. 2009

\begin{tabular}{|c|c|c|c|}
\hline Características & NAC(-)pH1N1* & $\mathrm{NAC}(+) \mathrm{pH} 1 \mathrm{~N} 1^{* *}$ & valor $p$ \\
\hline Leucocitos___mediana (RIQ), por $\mathrm{mm}^{3}$ & $9000(6500-13600)$ & $9450(6000-12775)$ & 0,992 \\
\hline Linfocitos__ mediana (RIQ), por $\mathrm{mm}^{3}$ & $2620(1680-4550)$ & $2009(1177-4389)$ & 0,066 \\
\hline Linfopenia__ $n(\%)$ & $30(34,5)$ & $33(66,0)$ & 0,000 \\
\hline Plaquetas__ conteo por $\mathrm{mm}^{3}$ mediana $(\mathrm{x} 1000)$ & $351(300-441)$ & $350(300-403)$ & 0,347 \\
\hline Hematocrito__ $\%$ media (rango) & $31,3(20-42)$ & $32.5(21-43)$ & 0,255 \\
\hline Proteína $\mathrm{C}$ reactiva__mediana (RIQ) & $7,2(0-40,1)$ & $6(0-38)$ & 0,433 \\
\hline$>10 \mathrm{mg} / \mathrm{l} \_\mathrm{n}^{\circ}(\%)$ & $16(18,4)$ & $13(26,0)$ & 0,294 \\
\hline Lactato deshidrogenasa__mediana (RIQ) U/l. & $1084(169-5957)$ & $1106(31-5957)$ & 0,924 \\
\hline Creatinina $(\mathrm{mg} / \mathrm{ml}) \_$mediana & $0,46(0,40-0,56)$ & $0,5(0,43-0,57)$ & 0,078 \\
\hline $\operatorname{Glucosa}(\mathrm{mg} / \mathrm{ml}) \_$mediana & $101(92-196)$ & $104(87-121)$ & 0,964 \\
\hline
\end{tabular}




\section{Fallecidos por virus de Influenza AH1N1/2009 pandémico}

Con respecto a los 12 casos fallecidos, 5 son NAC (+) pH1N1 y 7 son NNpH1N1. Todos con condiciones pre existentes; mediante el análisis de riesgo se encontró que ser un caso $\mathrm{NNpH1N} 1$ tiene alto riesgo de fallecer entre los casos neumonía por $\mathrm{pH} 1 \mathrm{~N} 1$, con un OR de 4,7 (IC: 1,1-20,1, p=0,02), no se encontró asociación entre el uso de oseltamivir, antecedente de asma/soba o el nivel de la PCR.

\section{Comparación entre los casos de NAC (+) pH1N1 y NAC (-) pH1N1}

Hallamos que es mayor la estadía hospitalaria en los casos de NAC $(+)$ pH1N1, el requerimiento de ventilación mecánica es más frecuente en los NAC (-) pH1N1, sin embargo esta diferencia es marginal (tabla $\left.\mathrm{N}^{\circ} 2\right)$. Con respecto a los síntomas, signos y exámenes de laboratorios se encontró asociación con la fiebre $(\mathrm{p}=0,009)$ y linfopenia $(\mathrm{p}<0,001)$ en los casos NAC $(+)$ pH1N1. No se encontró diferencia con la mortalidad entre ambos grupos (tabla $\left.\mathrm{N}^{\circ} 3, \mathrm{~N}^{\circ} 4\right)$.

\section{CONCLUSIONES Y DISCUSIONES}

Los hallazgos del estudio demuestran que la NAC en niños hospitalizados positivos y negativos al pH1N1 en tiempos de pandemia, son muy parecidos de acuerdo a los criterios clínicos, de laboratorio y mortalidad, siendo más frecuentes los menores de 3 años con condiciones pre existentes, información que debe precisarse que es obtenida de un hospital de referencia nacional como es el INSN, que atienden en su mayoría a niños con enfermedades pre existentes.

La pandemia del virus pH1N1 ocasionó en el INSN casos de $\mathbf{N N p H 1 N 1 , ~ o r i g i n a d o ~ a c c i o n e s ~}$ de investigación epidemiológicas y reacomodación de los servicios de hospitalización, por lo que sugerimos una búsqueda activa de casos, no solo en los hospitalizados, sino también en los trabajadores de salud $(17,18)$.

El 36\% de los casos de NAC (+) pH1N1 presentaron al menos una condición pre existente, muy parecido a otros estudios que abarcan de $14 \%$ a $75 \%$ (19-22); lo que demuestra que es adecuadas considerar grupo de riesgo de severidad a las niños previamente enfermos; si consideramos también que todos los que fallecieron tuvieron alguna condición pre existente. Sin embargo los niños NAC (-) pH1N1 presentaron 2 veces más casos de niños con condiciones pre existente, lo que indicaría que esta condición es importante en la mortalidad en niños con NAC independiente si presentan la infección por $\mathrm{pH} 1 \mathrm{~N} 1$ (2325).

El MINSA encargada de la vigilancia nacional de todos los casos de pH1N1 reportó que para ese periodo de estudio, los niños mayores de 2 años son los más afectados y que a medida que se incrementa la edad se incrementan el número de casos (10), a diferencia de los niños que requirieron hospitalización la mayor frecuencia de casos tanto en las $\mathrm{NAC}(+) \mathbf{p H} \mathbf{1 N} 1$ como en las NAC(-)pH1N1 fueron más los niños menores de 2 años, al igual que en otros estudios (26-29); Sin embargo los casos nosocomiales, son más frecuentes los mayores de 6 años.

Se evidenció que los casos NAC (+) pH1N1 al momento de su ingreso por emergencia, presentaron hipoxemia (saturación $0_{2}$ menos del 90\%) con el $32 \%(16 / 50)$, porcentaje parecido a los casos NAC (+) pH1N1 que fueron 33\% muy parecidos a otros estudios (26-28). Información importante para implementar con saturómetro los locales de diferentes niveles de atención.

En los casos severos de NAC(+)pH1N1 no se pudo hallar relación con la administración de oseltamivir, sugerimos realizar otros estudios 
prospectivos que demuestren el beneficio del antiviral (30).

Los síntomas gastrointestinales en los casos NAC (+) pH1N1 como son la diarrea y el vómito, considerados como importantes en el diagnostico según guía de la OMS y directiva sanitarias del MINSA, sin embargo creemos que estos síntomas no ayudan a nivel hospitalario en la diferenciación con las NACV (-) pH1N1, probablemente porque la mayoría de los niños son menores de 3 años, siendo los síntomas ambiguos y varían entre los examinadores por lo que creemos que se necesita de marcadores de severidad como cualquier otra neumonía $(31,32)$

Nosotros hayamos que la linfopenia pueden apoyar a la diferenciación de NAC (+) pH1N1 de los NAC (-) pH1N1, característica hematológica relacionado con pacientes con neumonía severa (17), por lo que deberían considerarse para sospechar los casos graves.

No se encontró diferencia en la mortalidad entre los casos NAC (+) pH1N1 y los casos NAC (-) pH1N1, iguales resultados son reportados en otros estudios $(33,34)$, es más, nosotros hayamos que los fallecidos con NAC (-) pH1N1 fueron más frecuentes, por lo que sugerimos ante la sospecha de un incremento significativo de neumonías severa identificar el agente etiológico y realizar una vigilancia epidemiológica adecuada. Siendo esto primordial si se trata de hospitales pediátricos o de referencia (35); así, obtendríamos información importante en detección oportuna de brotes e iniciar medidas adecuadas de control.

Creemos que en situación de epidemia o brote de alguna infección viral respiratoria, considerar la vacunación a los niños hospitalizados por tener un riesgo elevado a fallecer, también en el personal de salud, así como brindar el apoyo logístico en las medidas de control y prevención como el uso de alcohol gel, uso de mascarillas para evitar la transmisión. Nosotros creemos que la trasmisión de un virus respiratorio es mayor a nivel hospitalario que en la comunidad por la circulación de personas enfermas como son los padres de los pacientes, las familias y el personal de salud.

En conclusión. Los resultados de este estudio mostraría que los signos, síntomas y exámenes de laboratorio ayudan muy poco en la diferenciación de la NAC $(+)$ pH1N1 con las otras causas de neumonía; existe un mayor riesgo de mortalidad en los casos de NNpH1N1; el PCR elevado al ingreso y la presentación de condición preexistente están asociados a los casos severos de NAC (+) $\mathrm{pH} 1 \mathrm{~N} 1$.

\section{Conflictos de Interés}

Los autores declaran no tener conflictos de interés en la publicación de este artículo.

\section{REFERENCIAS BIBLIOGRÁFICAS}

1. Chan M. World now at the start of 2009 influenza pandemic. World Health Organization [in internet] 11 junio 2009 [accesado en junio 2010] Available in: http://www.who.int.

2. Dawood FS, Jain S, Finelli L, Shaw MW, Lindstrom $S$, Garten RJ, et al. Emergence of a novel swineorigin influenza A (H1N1) virus in humans. N Engl J Med. 2009 Jun 18;360(25):2605-15.

3. Childs RA, Palma AS, Wharton S, Matrosovich T, Liu Y, Chai W, et al. Receptor-binding specificity of pandemic influenza A (H1N1) 2009 virus determined by carbohydrate microarray. Nat Biotechnol. 2009 Sep;27(9):797-9.

4. Bermejo-Martin JF, Kelvin DJ, Eiros JM, Castrodeza J, Ortiz de Lejarazu R. Macrolides for the treatment of severe respiratory illness caused by novel H1N1 swine influenza viral strains. J Infect Dev Ctries. 2009;3(3):159-61.

5. Sherman SE, Foster J, Vaid S. Emergency Use Authority and 2009 H1N1 influenza. Biosecur Bioterror. 2009 Sep;7(3):245-50. 
6. Update: novel influenza $\mathrm{A}(\mathrm{H} 1 \mathrm{N1})$ virus infection - Mexico, March-May, 2009. MMWR Morb Mortal Wkly Rep. 2009 Jun 5;58(21):585-9.

7. Scalera NM, Mossad SB. The first pandemic of the 21st century: a review of the 2009 pandemic variant influenza A (H1N1) virus. Postgrad Med. 2009 Sep;121(5):43-7.

8. WHO. World Health Organization, Pandemic influenza preparedness and response: a WHO guidance document. Switzerland: WHO Library Cataloguing-in-Publication Data; 2009.

9. Ministry of Health. Plan nacional de preparación y respuesta frente a una potencial pandemia de influenza. Lima, Peru: Ministry of Health [in internet] Octubre 2005 [accesado en junio 2010] Available in: http://www.dge.gob.pe.

10. Munayco CV, Gomez J, Laguna-Torres VA, Arrasco J, Kochel TJ, Fiestas V, et al. Epidemiological and transmissibility analysis of influenza $\mathrm{A}(\mathrm{H} 1 \mathrm{~N} 1) \mathrm{v}$ in a southern hemisphere setting: Peru. Euro Surveill. 2009; 14(32).

11. Libster R, Bugna J, Coviello S, Hijano DR, Dunaiewsky M, Reynoso N, et al. Pediatric hospitalizations associated with 2009 pandemic influenza A (H1N1) in Argentina. N Engl J Med. Jan 7;362(1):45-55.

12. Neurologic complications associated with novel influenza A (H1N1) virus infection in children Dallas, Texas, May 2009. MMWR Morb Mortal Wkly Rep. 2009 Jul 24;58(28):773-8.

13. O'Riordan S, Barton M, Yau Y, Read SE, Allen U, Tran D. Risk factors and outcomes among children admitted to hospital with pandemic H1N1 influenza. CMAJ. 2010 Jan 12;182(1):39-44.

14. Zarychanski R, Stuart TL, Kumar A, Doucette S, Elliott L, Kettner J, et al. Correlates of severe disease in patients with 2009 pandemic influenza (H1N1) virus infection. CMAJ. 2010 Feb 23;182(3):25764.

15. Yu H, Zhang GH, Hua RH, Zhang Q, Liu TQ, Liao $\mathrm{M}$, et al. Isolation and genetic analysis of human origin $\mathrm{H} 1 \mathrm{~N} 1$ and $\mathrm{H} 3 \mathrm{~N} 2$ influenza viruses from pigs in China. Biochem Biophys Res Commun. 2007 Apr 27;356(1):91-6.
16. Miranda-Choque E, Ramirez C, Candela-Herrera J, Diaz J, Fernandez A, Kolevic L, et al. [Children hospitalized with influenza pneumonia AH1N1/2009 pandemic in the INSN]. Rev Peru Med Exp Salud Publica. 2011 Dec;28(4):610-6.

17. Perez-Padilla R, de la Rosa-Zamboni D, Ponce de Leon S, Hernandez M, Quinones-Falconi F, Bautista E, et al. Pneumonia and respiratory failure from swine-origin influenza $\mathrm{A}(\mathrm{H} 1 \mathrm{~N} 1)$ in Mexico. N Engl J Med. 2009 Aug 13;361(7):680-9.

18. Wicker S, Rabenau HF, Bickel M, Wolf T, Brodt R, Brandt C, et al. [Novel influenza H1N1/2009: virus transmission among health care worker]. Dtsch Med Wochenschr. 2009 Nov;134(48):2443-6.

19. Udompornwattana $S$, Srajai K, Suwan P, Tangsathapornpong A, Wittawatmongkol O, Phongsamart $\mathrm{W}$, et al. The clinical features, risk of prolonged hospitalization and household infections of hospitalized children for pandemic 2009 influenza A (H1N1) virus infection in Thailand. J Med Assoc Thai. 2012 Mar;95(3):403-11.

20. Kinikar AA, Kulkarni RK, Valvi CT, Mave V, Gupte N, Khadse S, et al. Predictors of mortality in hospitalized children with pandemic H1N1 influenza 2009 in Pune, India. Indian J Pediatr. 2012 Apr;79(4):459-66.

21. Kumar S, Havens PL, Chusid MJ, Willoughby RE, Jr., Simpson P, Henrickson KJ. Clinical and epidemiologic characteristics of children hospitalized with 2009 pandemic H1N1 influenza A infection. Pediatr Infect Dis J. 2010 Jul;29(7):591-4.

22. Al Subaie SS, Al Saadi MA. Features associated with severe disease in hospitalized children with 2009 influenza A (H1N1) infection at a university hospital in Riyadh, Saudi Arabia. Ann Saudi Med. 2012 Jan-Feb;32(1):53-8.

23. Sehgal V, Sethi GR, Sachdev HP, Satyanarayana L. Predictors of mortality in subjects hospitalized with acute lower respiratory tract infections. Indian Pediatr. 1997 Mar;34(3):213-9.

24. Li F, Zhang YH, Shao L, Chen Q. [Predictive indices for critical condition for infants and young children with severe pneumonia]. Zhongguo Dang Dai Er Ke Za Zhi. 2012 Sep;14(9):664-6. 
25. Suwanjutha S, Ruangkanchanasetr S, Chantarojanasiri T, Hotrakitya S. Risk factors associated with morbidity and mortality of pneumonia in Thai children under 5 years. Southeast Asian J Trop Med Public Health. 1994 Mar;25(1):60-6.

26. Lister P, Reynolds F, Parslow R, Chan A, Cooper $\mathrm{M}$, Plunkett $\mathrm{A}$, et al. Swine-origin influenza virus $\mathrm{H} 1 \mathrm{~N} 1$, seasonal influenza virus, and critical illness in children. Lancet. 2009 Aug 22;374(9690):6057.

27. Fraser C, Donnelly CA, Cauchemez S, Hanage WP, Van Kerkhove MD, Hollingsworth TD, et al. Pandemic potential of a strain of influenza A (H1N1): early findings. Science. 2009 Jun 19;324(5934):1557-61.

28. Louie JK, Acosta M, Winter K, Jean C, Gavali S, Schechter R, et al. Factors associated with death or hospitalization due to pandemic 2009 influenza A(H1N1) infection in California. JAMA. 2009 Nov 4;302(17):1896-902.

29. Muhammad Ismail HI, Tan KK, Lee YL, Pau WS, Razali KA, Mohamed T, et al. Characteristics of children hospitalized for pandemic (H1N1) 2009, Malaysia. Emerg Infect Dis. 2011 Apr;17(4):70810.

30. Ling LM, Chow AL, Lye DC, Tan AS, Krishnan P, Cui L, et al. Effects of early oseltamivir therapy on viral shedding in 2009 pandemic influenza A (H1N1) virus infection. Clin Infect Dis. $2010 \mathrm{Apr}$ 1;50(7):963-9.

31. Ramachandran P, Nedunchelian K, Vengatesan A, Suresh S. Risk factors for mortality in community acquired pneumonia among children aged 1-59 months admitted in a referral hospital. Indian Pediatr. 2012 Nov 8;49(11):889-95.

32. Tiewsoh K, Lodha R, Pandey RM, Broor S, Kalaivani M, Kabra SK. Factors determining the outcome of children hospitalized with severe pneumonia. BMC Pediatr. 2009;9:15.

33. Ostovar GA, Rubin LG, Rajan S, Sood SK, Kohn $\mathrm{N}$. Comparison of the clinical features of children hospitalized with pandemic 2009 A:H1N1 and seasonal influenza. Clin Pediatr (Phila). 2011 Apr;50(4):348-54.
34. Lera Carballo E, Worner NT, Sancosmed Ron M, Fabregas Martori A, Casquero Cossio A, Cebrian Rubio R. [Impact of the 2009 influenza A (H1N1) virus pandemic on the emergency department of a tertiary hospital.]. An Pediatr (Barc). 2011 Jul;75(1):13-20.

35. Laguna-Torres VA, Gomez J, Aguilar PV, Ampuero JS, Munayco C, Ocana V, et al. Changes in the viral distribution pattern after the appearance of the novel influenza A H1N1 ( $\mathrm{pH} 1 \mathrm{~N} 1)$ virus in influenza-like illness patients in Peru. PLoS One.5(7):e11719. 